\title{
Controlled Vesicle Self-Assembly in Continuous Two Phase Flow Microfluidic Channels
}

\author{
Andreas Jahn, Wyatt N. Vreeland, Laurie E. Locascio, Michael Gaitan \\ Semiconductor Electronics Division and Analytical Chemistry Division \\ National Institute of Standards and Technology \\ Gaithersburg, MD 20899
}

\begin{abstract}
This paper describes a method to form monodisperse populations of liposomes in a continuous 2-phase flow microfluidic network with precision control of their size from $100 \mathrm{~nm}$ to $300 \mathrm{~nm}$ by manipulation of the fluid flow rates. We demonstrate that water-soluble reagents can be encapsulated in the liposomes enabling formulations for therapeutic or drug delivery applications. We conclude that by creating a solvent-aqueous interfacial region in a microfluidic format that is homogenous and controllable on the length scale of a nanoparticle self-assembly process, we can facilitate the fine control of its size and polydispersity.
\end{abstract}

\section{INTRODUCTION}

Liposomes [1] are one example of nanoparticles that have been used for a wide variety of medical applications including targeted drug delivery and DNA transfection [2,3]. Liposomes are cellular mimetics composed of a lipid bilayer membrane that encapsulates and sequesters species inside from species residing outside the membrane. Traditional liposome preparation methods $[4,5,6,7,8,9,10,11,12,13]$ are all conducted through mixing of bulk phases, leading to inhomogeneous chemical and/or mechanical conditions during liposome formation; hence liposomes are often polydisperse in size and lamellarity. Liposome size and size distribution are important for in vivo applications where size ultimately determines drug dosage. The traditional methods to formulate liposomes require additional steps such as membrane extrusion or sonication in order to yield the desired homogeneous liposome populations. Methods that can control liposome size during formation without the need for additional steps would be of great benefit. Here we report on a method that shows promise towards this goal.

\section{METHOD}

In our approach to liposome formation, we employ the laminar characteristics of fluidic flow in a micrometer-scale channel to precisely control the distribution of chemical conditions and mechanical forces so that they are constant on a length scale equivalent to that of the liposome. We find that forming liposomes in a micrometer-scale flow field results in more homogenous conditions during liposome self-assembly and the resultant liposome populations are more uniform in size, and hence of low polydispersity. A schematic diagram of the microfluidic device is shown in Figure 1. We hydrodynamically focus [14] an isopropyl alcohol solution containing dissolved phospholipids [15] (center inlet channel) at a microchannel cross-junction between two aqueous buffer streams (side inlet channels).

When the lipid solution comes into contact with the aqueous solution, the isopropyl alcohol rapidly diffuses into the aqueous phase and vice versa. Diffusive mixing typically requires a

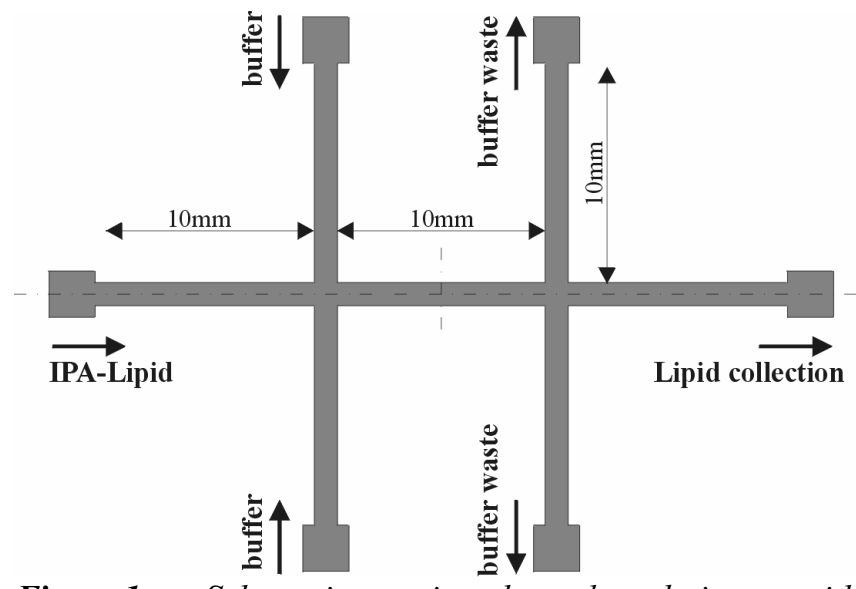

Figure 1. Schematic microchannel design with corresponding fluid flows; microchannels have trapezoidal cross-section; maximum width is $200 \mu \mathrm{m}$, minimum width is $146 \mu \mathrm{m}$ and depth is $40 \mu \mathrm{m}$.

substantial amount of time and space to complete. However, fast mixing can be obtained by reducing the length scale over which the fluid must diffusively mix by hydrodynamically focusing the injection stream. The flow rates of the isopropyl alcohol and buffer channels are adjusted to control the degree of hydrodynamic focusing, which affects the chemical and mechanical conditions at the lipid-buffer interface and ultimately the liposome size. The lipids assemble into liposomes when the alcohol is diluted to a concentration where lipids are no longer soluble. The focusing stream width can be controlled by varying the flow ratios between the sample and buffer, as can be seen in Figure 2.

Immediately downstream of the first cross intersection, the fluorescent intensity of the center stream containing phospholipids increases sharply indicating the formation of the lipids into liposomes. This effect is due to an increased quantum efficiency of the fluorescent dye used in this experiment $\left(\mathrm{DiIC}_{18}\right)$ upon incorporation into a lipid membrane. This effect is most dramatically illustrated in Figure 3.

After the formation process is completed at the fist cross intersection, the liposomes continue to flow along the stream to the second cross intersection in a tightly focused stream, owing to the low Reynold's number laminar flow typical of microfluidics and their comparatively low diffusion coefficient. At this second cross intersection, excess buffer is removed via the two side outlet channels and the liposome suspension is collected via the center outlet channel. The liposomes form (as demonstrated by increased fluorescence of the $\mathrm{DiIC}_{18}$ ) along the boundary between the isopropyl alcohol and water. At all flow rates tested, a ridge of increased fluorescence is clearly visible as the aqueous streams focus the isopropyl alcohol solution. Fluorescence intensity increases to its maximum value immediately downstream of the minimum width of the isopropyl alcohol stream indicating the highest concentration of liposomes. 

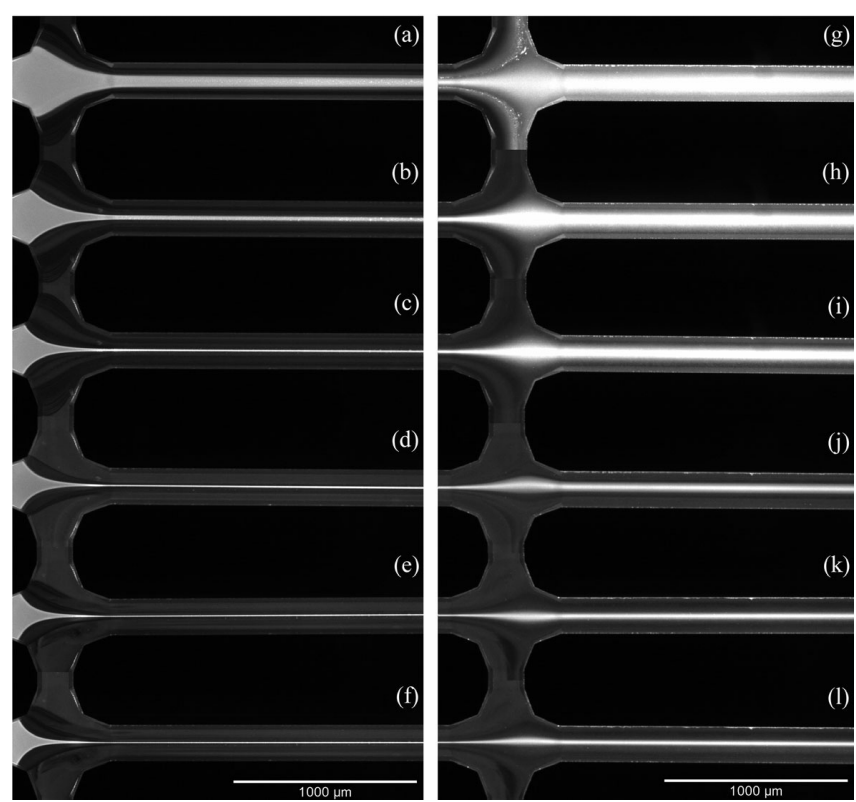

Figure 2. Fluorescent images of DiIC $C_{18}$ in IPA-lipid mixture at different flow ratios; (a)-(f) shows the focusing of IPA at the first intersection at different volumetric flow rates. IPAlipid mixture fed in from the left into the middle channel at constant volumetric flow rate of $0.7 \mu \mathrm{l} \mathrm{min}{ }^{-1}$; PBS is fed in from the side inlets at volumetric flow rates of (a) 1, (b) 5 , (c) 10, (d) 15 , (e) 20 and (f) $25 \mu \mathrm{min}^{-1}$, respectively; images (g)-(l) show the variation of the IPA-lipid stream at the second intersection at the flow rates according to (a)-(f). The silicon/glass microchannels have trapezoidal cross sections with the following dimensions: depth $=40 \mu \mathrm{m}$, maximum width $=200 \mu \mathrm{m}$, minimum width $=146 \mu \mathrm{m}$.

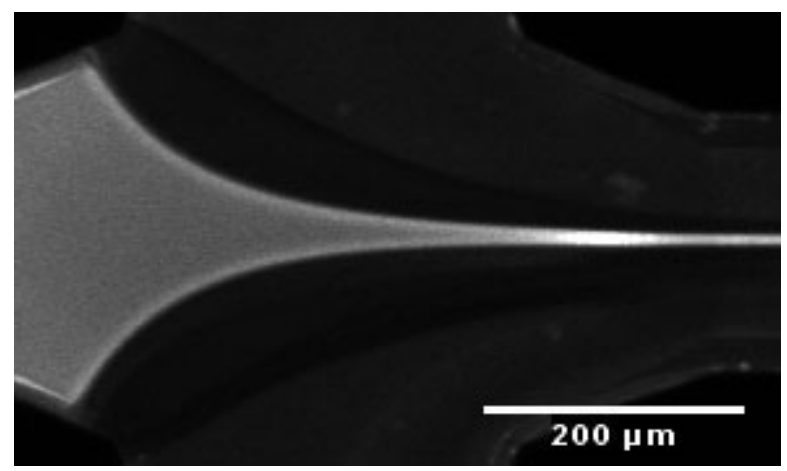

Figure 3. Magnified view of the injection stream of Figure $2 d$ showing increased fluorescence of the DiIC ${ }_{18}$ due to its increased quantum efficiency upon incorporation into a lipid membrane.

\section{DEVICE FABRICATION}

The device consists of a silicon wafer containing the microfluidic structures, a borofloat glass wafer (BSG) to seal the microchannels and fluidic connectors to inject fluid through (a)

(b)

(c)

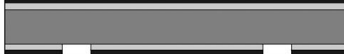

(d)

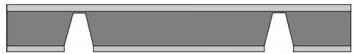

(e)

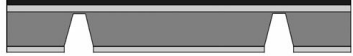

(f)

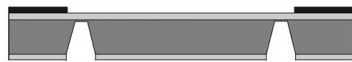

(g)

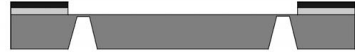

(h)

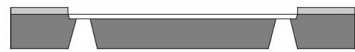

(i)

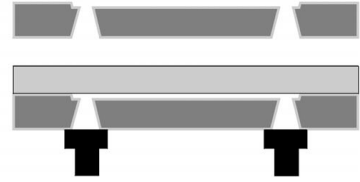

Silicon dioxide

$\square \quad$ Silicon

Photoresist

Figure 4. Fabrication process flow; drawing not to scale.

syringes via PEEK capillary tubing. Figure 4 shows the details of the fabrication process flow.

A layer of $1.3 \mu \mathrm{m}$ thick positive resist (MicroChem S1813) is spin-coated and patterned on the back side of an oxidized silicon wafer followed by an HF dip to pattern the exposed oxide. Fluidic through-holes are then partially etched anisotropically through the back side using 2:1 mixture of $\mathrm{H}_{2} \mathrm{O}$ and $25 \%$ tetramethylammoniumhydroxide (TMAH) heated to $80^{\circ} \mathrm{C}$ leaving a thin silicon membrane of approximately $20 \mu \mathrm{m}$ thickness. Afterwards, the oxide on the front side of the silicon wafer is patterned with a microfluidic network of $200 \mu \mathrm{m}$ wide and $40 \mu \mathrm{m}$ deep microchannels that are anisotropically etched using the same TMAH process. The oxide is removed completely and the wafer reoxidized to form a homogenous surface for the following anodic bonding process.

Prior to bonding, the glass wafer $(75 \mathrm{~mm}$ diameter, $0.1 \mathrm{~mm}$ thick, Corning Pyrex 7740) and the silicon wafer are thoroughly cleaned and dehydrated to ensure intimate contact between the wafers. The wafers are then anodically bonded at $400{ }^{\circ} \mathrm{C}$ for 60 min with a ramp rate of $25^{\circ} \mathrm{C} \mathrm{min}^{-1}$ and a bonding voltage of $800 \mathrm{~V}$. The thermal expansion coefficient (TEC) of borofloat glass (BSG) is well matched to that of silicon below $400{ }^{\circ} \mathrm{C}$ which greatly reduces the stress of the microchannel network device.

We use fluidic nanoports (F-124S, Upchurch Scientific, USA) for our fluidic connections between capillary tubing and the microchannel network in order to minimize volume to the fluid path. The nanoports are bonded to the backside of the silicon wafer using adhesive rings that are provided with the nanoports by clamping them to the wafer between two metal plates and curing them in a convection oven at $121^{\circ} \mathrm{C}$ for $90 \mathrm{~min}$. PEEK ${ }^{\mathrm{TM}}$ capillary tubes deliver the fluid from glass syringes to the microchannel network. 


\section{EXPERIMENTAL DETAILS}

Reagent transfers were obtained using gastight syringes interfaced to the microchannel network through capillary tubing (PEEK Tubing, Upchurch Scientific) and capillary connectors (Nanoports, Upchurch Scientific) that were bonded to the fluid through-holes etched in the silicon wafer. Two programmable high accuracy syringe pumps PHD 2000 from Harvard Apparatus were used to inject sample and buffer solution into the microchannel. A Labview software interface ensured the precise control of the sample and buffer flowrates. Sample consumption of the lipid solution was on the order of $5-15 \mathrm{nl} \mathrm{s}^{-1}$ and experiments were performed with as little sample as $100 \mu 1$.

Dimyristoylphosphatidylcholine (DMPC) and cholesterol in a molar ratio of 1:1 were diluted in chloroform (all from Aldrich) with $1 \mathrm{wt} \%$ of 1,1 '-dioctadecyl-3,3,3',3'-tetramethilindocarbocyanine perchlorate $\left(\mathrm{DiIC}_{18}\right)$ added for fluorescent imaging. The chloroform solvent was evaporated under a stream of nitrogen at room temperature to form a lipid film on the bottom of a test tube. The test tube was then placed in a vacuum desiccator for at least $24 \mathrm{~h}$ to ensure dryness. The dried lipid mixture was resolubilized with $500 \mu \mathrm{l}$ dry isopropyl alcohol yielding a $10 \mathrm{mM}$ concentration of lipid solution. Phosphate buffered saline solution $(10 \mathrm{mM}$ phosphate, $27 \mathrm{mM}$ potassium chloride, $137 \mathrm{mM}$ sodium chloride, $\mathrm{pH}=7.4$ ) was used as a hydration buffer. To demonstrate the encapsulation process, a fluorescent dye, carboxyfluorescein $(\mathrm{CF})$, was incorporated into the buffer stream at a concentration of $1 \mathrm{mM}$. Upon formation of the liposomes at the interface between isopropyl alcohol and buffer solution, the liposomes encapsulated a discrete volume of buffer, and with that entrapped dissolved CF. The liposome formulations were collected at each flow condition in polycarbonate cuvets. After collection, phosphate buffered saline solution $(10 \mathrm{mM})$ was added to each formulation and sealed for further characterization.

The flow profile in the microchannel were imaged with a fluorescence microscope (Axioplan 2, Carl Zeiss, Thornwood, NY, USA) using a halogen lamp as an excitation source with appropriate filters for excitation and detection of $\mathrm{DiIC}_{18}$ (excitation $540 \mathrm{~nm} \pm 12.5 \mathrm{~nm}$; beam splitter $565 \mathrm{~nm}$; emission $605 \pm 27.5 \mathrm{~nm}$ ) and carboxyfluorescein (excitation $470 \mathrm{~nm} \pm 20 \mathrm{~nm}$; beam splitter $510 \mathrm{~nm}$; emission $515 \mathrm{~nm}$ ) and digitalized using a high sensitivity Hamamatsu Photonics Orca-ER CCD camera.

The size of the liposomes were determined at $21{ }^{\circ} \mathrm{C}$ by quasi elastic light scattering (QELS) with a Nanosizer (Coulter ${ }^{\mathbb{B}}$ N4MD, Coulter Electronics, USA). Samples containing 100-200 $\mu \mathrm{l}$ of liposome solutions were collected in polycarbonate cuvets at the lipid collection port of the microchannel and diluted with $1 \mathrm{~mL}$ of $10 \mathrm{mM}$ buffered saline solution. The cuvets were then placed into the sample compartment of the light scattering device. Five measurements were taken and averaged for each sample (Figure 5).

Cryo transmission electron microscopy (Cryo-TEM) involves the examination of a vitrified hydrated sample directly on a cryo stage in the TEM. Five microliter drops of liposome suspensions were placed on 1000-mesh copper EM grid. Specimens were frozen by clamping each grid into spring-loaded forceps of a Leica KF80 freezing machine. The grid was blotted with filter paper to leave a thin film of liposome suspension just prior to plunging the grid into liquid ethane cooled to $-180^{\circ} \mathrm{C}$ by liquid nitrogen. The frozen grid was then loaded under liquid nitrogen into a Gatan model 626 specimen holder and cryotransferred into an FEI CM120 transmission electron microscope (Philips) equipped with Gatan anticontaminator blades. Suitably thin specimen regions were imaged at a beam voltage of $120 \mathrm{kV}$ and at an electron dose of less than 1000 electrons per square nanometer using a Gatan GIF100 post-column imaging filter equipped with a $1024 \times 1024$ pixel cooled CCD camera. Contrast was enhanced by energy-filtering the transmitted electrons and by underfocusing the objective lens to about $500 \mathrm{~nm}$.

The main advantage here is that no other preparation is necessary, and the presence of unwanted ice crystals is easily detected. The liposome diameter of approximately $100 \mathrm{~nm}$ observed with the cryo-TEM (Figure 6) was in agreement with that determined by quasi-elastic light scattering $(95 \mathrm{~nm} \pm 30 \mathrm{~nm})$.

\begin{tabular}{|c|c|c|}
\hline $\begin{array}{c}\text { flow ratio } \\
/ 1 /\end{array}$ & $\begin{array}{c}\text { mean diameter } \\
/ \mathrm{nm} /\end{array}$ & $\begin{array}{c}1 \sigma \\
/ \mathrm{nm} /\end{array}$ \\
\hline 2 & 233 & 68 \\
10 & 207 & 46 \\
20 & 150 & 40 \\
30 & 106 & 28 \\
40 & 100 & 20 \\
\hline
\end{tabular}

Figure 5. Liposome size and standard deviation $(\sigma)$ vs. different flow ratios. IPA-Lipid mixture is injected at a constant volumetric flowrate of $1.0 \mu \mathrm{min}^{-1}$. Flow ratio is defined as the center flowrate to the total aqueous flowrate from the two side channels.

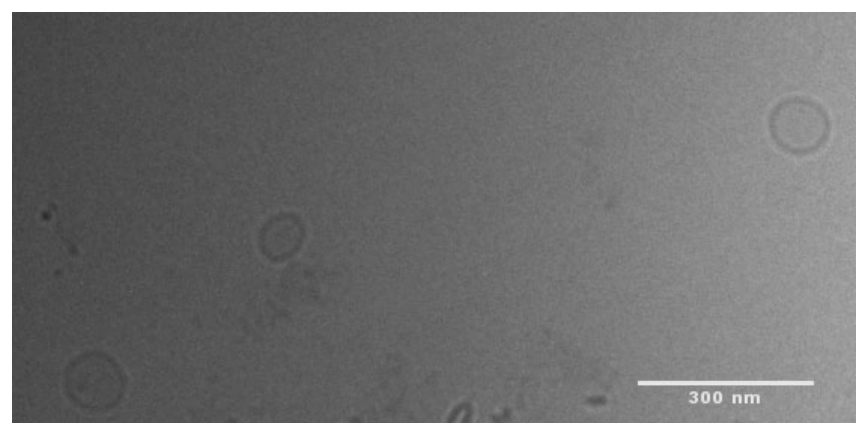

Figure 6. Cryo-TEM of unilamellar liposomes at $120 \mathrm{kV}$ beam voltage and less than 1000 electrons per square nanometer electron dose. The image shows unilamellar liposomes produced by this method.

\section{CONCLUSION}

By changing the length scale of the fluidics in which lipids self-assemble into liposomes and simultaneously manipulating both the length scale and the shear forces applied to the vesicles upon formation, we have achieved fine control of liposome size and homogeneity. Microfluidics allows us to adjust the flow fields precisely using the simple principle of hydrodynamic focusing. We anticipate that this method could be used to manufacture liposome formulations on demand and thus avoid issues of poor shelf life that have been problematic with other processes.

\section{ACKNOWLEDGEMENTS}

We gratefully acknowledge Dr. M. Richter of the Fraunhofer Institute IZM for supporting the guest researcher opportunity for A.J. at NIST. We also acknowledge the NIST/NRC Postdoctoral 
Associate Program for support of W.N.V. and funding from the NIST Director's Single Molecule Manipulation and Measurement Program. We also acknowledge Dr. Richard Leapman at NIH for his support with the cryo transmission electron microscopy imaging.

\section{REFERENCES}

1. A. D. Bangham, M. M. Standish, J. C. Watkins, "Diffusion of Univalent Ions Across the Lamellae of Swollen Phospholipids", $J$. Mol. Biol. 13, 238-253 (1965).

2. G. Gregoriadis, "Liposome Technology Volume 3; Targeted Drug Delivery and Biological Interactions", (CRC Press, Boca Raton, 1983).

3. D. D. Lasic, D. Papahadjopoulos, "Liposomes Revisited", Science 267, 1275-1276 (1995).

4. G. Gregoriadis, H. da Silva, A. T. Florence, "A Procedure for the Efficient Entrapment of Drugs in Dehydrationrehydration Liposomes (DRVs)", Int. J. Pharm. 65, 235-242 (1990).

5. F. C. Szoka, D. Papahadjopoulos, "Procedure for Preparation of Liposomes with Large Internal Aqueous Space and High Capture by Reverse Phase Evaporation", Proc. Natl. Acad. Sci. U.S.A. 75, 4194-4198 (1978).

6. C. Pidgeon, S. McNeely, T. Schmidt, "Multilayered Vesicles Prepared by Reverse Phase Evaporation: Liposome Structure and Optimum Solute Entrapment", Biochemistry 26, 17-29 (1987).

7. H. Hauser, "The Effect of Ultrasonic Irradiation on the Chemical Structure of Egg Lecithin", Biochim. Biophys. Res. Commun. 45, 1049-1055 (1971).

8. S. Batzri, E. D. Korn, "Single Bilayer Liposomes Prepared without Sonication", Biochem. Biophys. Acta. 298, 1015-1019 (1973).

9. T. H. Fischer, D. D. Lasic, " A Detergent Depletion Technique for the Preparation of Small Vesicles", Mol. Cryst. Liq. Cryst. Lett. 102, 141-145 (1984).

10. H. Kikuchi, H. Yamauchi, S. Hirota, "A Spray-Drying Method for Mass-Production of Liposomes", Chem. Pharm. Bull. 39, 1522-1527(1991).

11. A. Wagner, K. Uhl-Vorauer, G. Kreismayer, "The Crossflow Injection Technique - an Improvement of the Ethanol Injection Method", J. Lip. Res. 12, 259-270 (2002).

12. T. S. Aurora, W. Li, H. Z. Cummins, "Preparation and Characterization of Monodisperse Unilamellar Phospholipid Vesicles with Selected Diameters from 300 to $600 \mathrm{~nm}$ ", Biochimica et Biophysica Acta 820, 250-258 (1985).

13. P. L. Luisi, P. Walde, "Giant Vesicles", (John Wiley \& Sons, Chichester, 2000).

14. J. B. Knight, A. Vishwanath, J. P. Brody, J. P., R. H. Austin, "Hydrodynamic Focusing on a Silicon Chip: Mixing Nanoliters in Microseconds", Phys. Rev. Lett. 80 (17), 3863-3866 (1998).

15. L. Locascio-Brown, A. L. Plant, V. Horvath, "Liposome Flow Injection Immunoassay: Implications for Sensitivity, Dynamic Range, and Antibody Regeneration”, Anal. Chem. 62, 2587-2593 (1990). 